\title{
Assessment of tripping hazard by a single step evaluated by principal component analysis of pedestrian feet movements and eye behaviours
}

\author{
Tatsuto Suzuki ${ }^{1}$, I Wa Liu ${ }^{2}$, Nikolaos Papadosifos ${ }^{1}$, Derrick Boampong ${ }^{1}$, \\ Pak Sum Fung ${ }^{2}$, and Nick Tyler ${ }^{2}$ \\ ${ }^{1}$ Pedestrian Accessibility Movement Environment Laboratory, Department of Civil, \\ Environment, Geomatic Engineering, University College London, London N19 5UN, UK \\ t.suzuki@ucl.ac.uk \\ ${ }^{2}$ Department of Civil, Environment, Geomatic Engineering, University College London, \\ London WC1E 6BT, UK
}

\begin{abstract}
The aims in this study was to investigate feet movements and eye behaviours of young and older people when stepping over a small single step up to $25 \mathrm{~mm}$ height while walking. In addition, this study investigated what factors in feet movements and eye behaviours can be used to show effectiveness of the preventive measures of tripping. Healthy 12 young and 5 older participants joined this study. A walkway ( $9.6 \mathrm{~m}$ length $\mathrm{x} 1.2 \mathrm{~m}$ width) with a single step chosen from 0,10 , and $25 \mathrm{~mm}$ in one of three positions $4.8,6$, and $7.2 \mathrm{~m}$ from the start line, was used for measurement of feet movements and eye behaviours in stepping over. Principal component analysis demonstrated to evaluate pedestrian capability of stepping over the single step and showed differences between young and older groups. First principal component might show different walking style, second principal component might show foot strategy of stepping over the single step, and third principal component might show that awareness of the single step. The awareness level of $25 \mathrm{~mm}$ step by older group was similar level of the awareness of $10 \mathrm{~mm}$ by young group, and older group was struggle to aware $10 \mathrm{~mm}$ step, compared with $0 \mathrm{~mm}$ case. These findings might be useful to consider how to improve outdoor and indoor environments as well as to assess how physical training, preventive measures, and assistive technologies to reduce the risk of tripping in stepping over the single step.
\end{abstract}

Keywords: Tripping, Single step, Feet movements, Eye behaviours, Principal component analysis.

\section{$1 \quad$ Introduction}

Small single steps can be seen in outside environments in daily life. Pedestrians walking in outside meet the edge of lifted block pavements caused by the raised ground, the edge of dropped pedestrian kerb, the edge of a train floor on the platform in level access, for example. UK footway maintenance policy basically guides to repair over 
$20 \mathrm{~mm}$ height level uneven part on footways, and leaves under $20 \mathrm{~mm}$ level untreated [1]. Pedestrians can detect such a small single step before approaching, and then step over it naturally, however, sometimes tripping happens for different reasons [1].

Tripping while walking is a major cause of falling [2], which sometimes leads serious injuries in people aged 65 years and over [3] because ageing slows counter actions of legs to recovery walking balance and of hands to protect a body from crushing to the ground. In addition, ageing slows cognitive process in brain. Tripping occurs when natural movement of a swing foot in walking was forced to limit by a contact with an object, the ground, or the single steps [4].

Previous studies with 3D marker measurement systems focused on minimum foot clearance in walking on flat surface [5] as well as feet movement when stepping over an obstacle [6,7], in order to investigate how pedestrians naturally prevent their feet movements from tripping on the ground or obstacles higher than $25 \mathrm{~mm}$. Recent study focused on cognitive process how pedestrians can detect an object or a step surface while walking [8,9]. Previous findings partly provide evidences how pedestrians naturally walk without tripping, however, no investigation with feet movements and eye behaviours together when stepping over a step has been done.

Meanwhile, coloured edge of steps, warning signs, reminders in the railway platform, increased floor illuminance, etc are used to prevent pedestrians from tripping. These preventive measures would reduce tripping hazard, however, it's difficult to expect how these measures are effective before implementation.

The aims in this study was to investigate feet movements and eye behaviours when stepping over a small single step up to $25 \mathrm{~mm}$ height while walking, in order to explore in more details how young and older pedestrians prevent from tripping. In addition, this study investigated what factors in feet movements and eye behaviours can be used to show effectiveness of the preventive measures of tripping.

\section{Methods}

\subsection{Experiment setup}

A walk way for this study in Figure 1 was situated in the middle of the platform in the Pedestrian Accessibility Movement Laboratory (PAMELA), University College London. The total distance of walkway was $9.6 \mathrm{~m}$ from a start line to an end line and its width was $1.2 \mathrm{~m}$. The size of the PAMELA platform was $12 \times 7.2 \mathrm{~m}$ and consisted of 60 modules having a $1.2 \times 1.2 \mathrm{~m}$ concrete surface on the top of each module. Each module could control height of the concrete surface by four actuators supporting each corner. The concrete surface of each module had nine $400 \times 400 \mathrm{~mm}$ square pavers. Both the start and end lines were marked by yellow black stripe $1.2 \times 0.05 \mathrm{~m}$. The height of a single step was chosen from 0,10 , and $25 \mathrm{~mm}$, and was created at one of three positions $4.8,6$, and $7.2 \mathrm{~m}$ from the start line. Only one single step was faced in each trial and the height between the single step and the end line was adjusted to the same level. The height and position of the single step was randomised for trials so that participants could not expect the position and height of the single step. Floor illuminance on the walkway was calibrated to 256lux by a LED lamp system fixed at $3 \mathrm{~m}$ height from the walkway. 


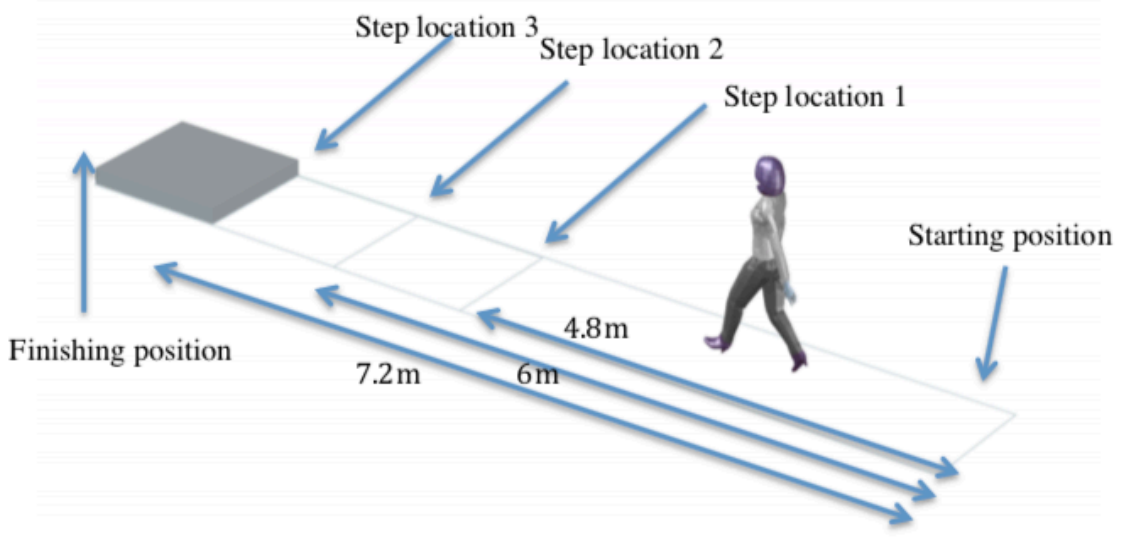

Fig. 1. Walkway with three different positions of a single step with height 0,10 , or $25 \mathrm{~mm}$. Only one single step was faced in each trial and the height between the single step and the end line was adjusted to the same level.

\subsection{Measurement system}

Active marker 3D measurement system by infrared LED (CODA system by CODAmotion - Charnwood Dynamics Ltd), which can measure marker 3D positions in $0.3 \mathrm{~mm}$ accuracy, was used to capture 3D positions of participant's feet and single step on the walkway. Two CODA cameras were placed after confirming enough area coverage to capture feet movements over three single step positions and were calibrated before trials. A pair of CODA makers were placed on both sides of the edge of each single step position on the walkway and four shoe positions (Toe, left, right, and heel) of both feet. Sampling frequency of CODA measurements were 100Hz. Participants wore mobile eye tracking glasses (SensoMotoric Instruments $\mathrm{GmbH}$ ), which measured the position and duration of fixations at $30 \mathrm{~Hz}$ sampling.

\subsection{Experiment protocol}

Before trials, an examiner had asked a participant to naturally walk to the end line of the walkway from the start line. Participants allowed free fixation and head movement while walking. The single step had been created by remote control before each trial while the participant waited with sitting on a chair behind of a screen, which blocked visual view of the participants to see the walkway. After finishing set up of the single step, the participant was asked to stand at the start line, and then, started walking to the end line after the examiner moved out the screen. When the participant reached the end line, each trial ended, and the participant was asked to come back and to wait for next trial with sitting on the chair in the back of the screen. 


\subsection{Participants}

Total 17 healthy participants without any visual, mobility and cognitive disorders, joined this study after providing written informed consent on the experiment protocol approved by the University College London Research Ethics Committee. Young group was formed by 12 participants (age $=25.9 \pm 7.6$ years, height $=1.66 \pm 0.07 \mathrm{~m}$, and body weight $=56.1 \pm 5.3 \mathrm{~kg}$ ), and older group was formed by five participants (age $=$ $69.3 \pm 8.6$ years, height $=1.56 \pm 0.05 \mathrm{~m}$, and body weight $=46.1 \pm 3.1 \mathrm{~kg}$ ).

\subsection{Data analyses}

With CODA measurements of both feet movements, a swing foot (SWF) stepping over the single step was identified in the beginning of analysis, and then stance foot (STF) was determined. Extracted parameters from SWF movement were vertical toe clearance from the edge of the single step, vertical maximum toe clearance from the ground, horizontal heel position from the edge of the single step after heal contact of SWF, mean SWF speed, and SWF step length. From STF movement, horizontal toe position from the edge of the single step was extracted. Eye tracking data were used for extracting total duration of fixations at the step, $\pm 1 \mathrm{~m}$ step area, and travel path at each trial, and these fixation durations were normalised by travel time. All extracted parameters were analysed with principal component analysis [10].

\section{Results}

Total variance explained of data difference by from first principal component (PC1) to third one (PC3) was $75.34 \%$. The Loadings of all parameters in the PC1 - 3 were shown in Fig. 2. PC1's main parameters were SWF step length (-0.48), SWF mean speed (-0.48), and travel time (0.45). PC2's main parameters were SWF maximum toe clearance $(0.58)$, SWF toe clearance $(-0.56)$, and STF toe position (0.55). Main parameters of PC 3 were duration of fixation at the single step (0.78), and SWF heel position (0.57). Loading of parameters provides how parameters individually contribute to PC scores, and the range of loadings is from -1 to 1 .

Mean PC scores grouped by groups and single step heights are shown with standard error of means in Fig. 3. PC1 showed large difference between young and old groups. Young group in three step height cases showed similar PC1 scores $-0.67[0 \mathrm{~mm}]$, $068[10 \mathrm{~mm}],-075[25 \mathrm{~mm}]$, and Older group showed larger PC1 scores $0.80[0 \mathrm{~mm}]$, $1.26[10 \mathrm{~mm}]$, and $1.32[25 \mathrm{~mm}]$. PC2 showed similarity between young and old with around zero, except $0.36[25 \mathrm{~mm}]$ by young group and $-0.61[10 \mathrm{~mm}]$ by older group. PC3 scores of both groups had a trend to increase by step height from similar base level at $0 \mathrm{~mm}$ step height. PC3 scores of young group were $-0.58[0 \mathrm{~mm}],-0.05[10 \mathrm{~mm}]$, and $0.57[25 \mathrm{~mm}]$. Older group PC3 scores were $-0.68[0 \mathrm{~mm}],-0.40[10 \mathrm{~mm}]$, and $0.04[25 \mathrm{~mm}]$.

All data distribution PC3 by PC1 is shown in Figure 4. Young group's PC scores were placed in left side plane, and young $25 \mathrm{~mm}$ cases moved to left upper area from 
left lower area. Older group PC scores were placed in right side place, and older $25 \mathrm{~mm}$ cases spread to left side area from lower middle area.

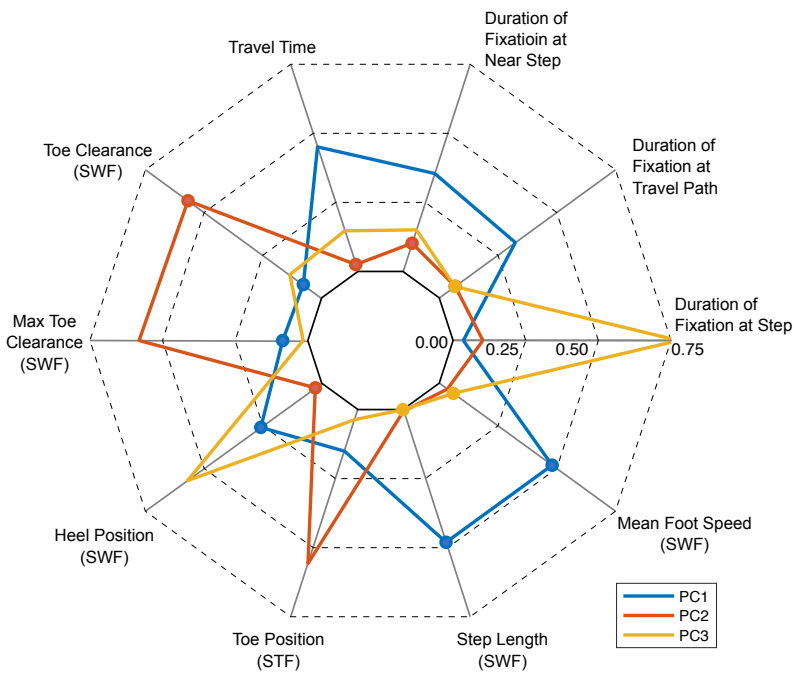

Fig. 2. Loading of all parameters of PC1 - PC3. Negative loadings are shown with a circle.

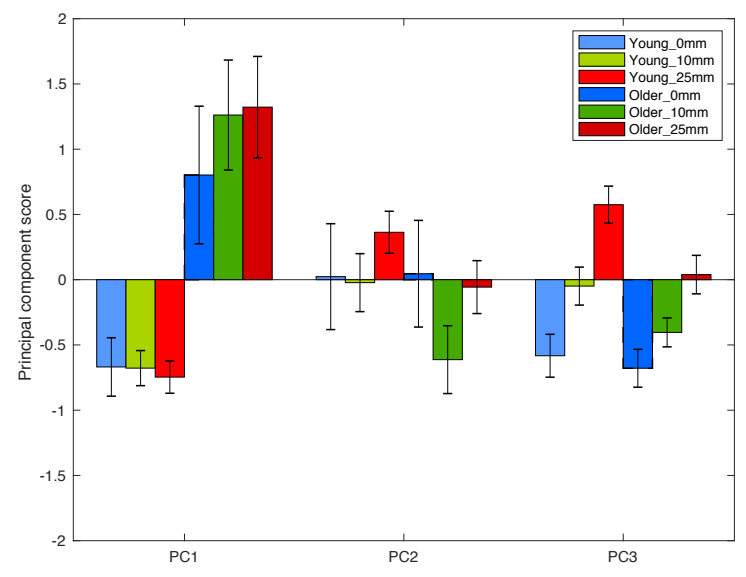

Fig. 3. Mean scores of PC1 to PC3 with standard error of means 


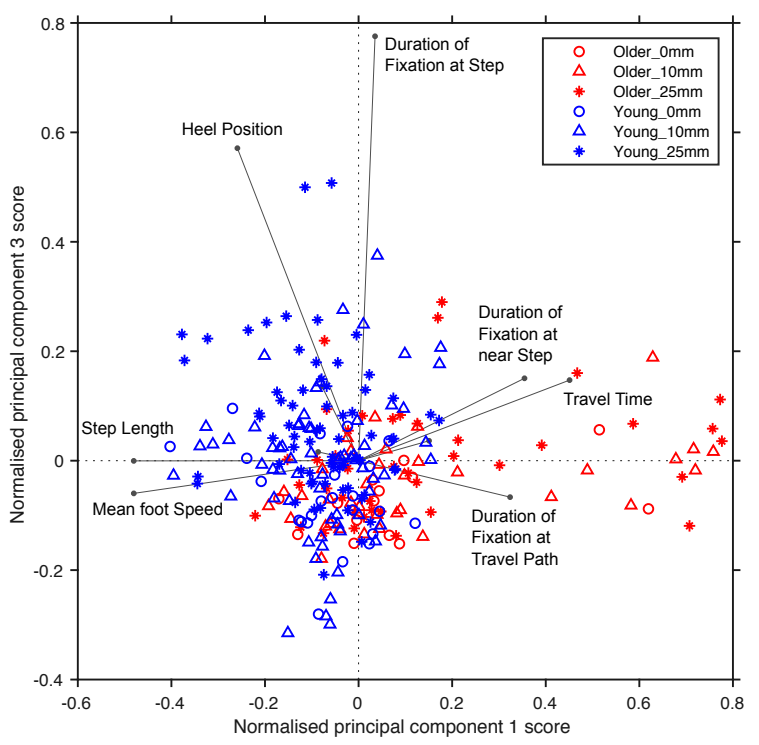

Fig. 4. Biplot of $\mathrm{PC} 1$ and $\mathrm{PC} 3$

\section{$4 \quad$ Discussions}

PC1 might mean individual walking style of young or older. PC1 score in Fig. 3 clearly shows difference between young and older groups. Older group took longer travel time caused by shorter step length and lower mean SWF speed with longer duration of fixation on near step and travel path. In opposite, younger group walking fast without looking on the ground. This might be because young group might detect the single step by peripheral vision. The step height didn't affect walking style of young group, but older group became more careful of walking on $25 \mathrm{~mm}$ case than $0 \mathrm{~mm}$.

PC2 might mean general foot strategy of stepping over the single step. SWF's Higher maximum toe clearance and lower toe clearance happened when horizontal STF toe position was near to the edge of the single step. A possible reason was that STF was placed near the single step in order to keep flexibility of SWF movement and SWF started moving near the edge of the step, so toe clearance was lowered and participants intentionally raised toe to avoid tripping. PC2 scores of both groups in Fig. 3 were similar, however, young group at $25 \mathrm{~mm}$ case used this strategy clearly compared with other cases, and older group didn't take this strategy in 10mm cases.

PC3 might mean that awareness of the single step. Longer duration of fixation at the single step contributes further horizontal SWF heel position after heel contact. The trend of PC3 by the step height in Fig. 3 possibly suggest that younger group showed increased awareness of the step by the increase of the step height proportionally, and older group's awareness of the single step $10 \mathrm{~mm}$ were slightly better than $0 \mathrm{~mm}$, but not a similar difference between 0 and $10 \mathrm{~mm}$ cases in young group. The results might say the awareness level of $25 \mathrm{~mm}$ step by older group was similar level of the awareness of 
$10 \mathrm{~mm}$ by young group, and older group struggled to aware $10 \mathrm{~mm}$ step, compared with $0 \mathrm{~mm}$ case.

In the biplot PC1-PC3 in Fig. 4, it might be possible to spot individual problem. These three PC scores showed typical manners and capability of stepping over a single step by young and older groups and can be used to evaluate tripping hazard and how preventive measures improve older people's manner and capability.

This study was carried out with limitations; 1 ) A black battery boxes $33 \times 51 \times 14 \mathrm{~mm}$ with black active markers $12 \times 8 \times 5 \mathrm{~mm}$ were visible on three single step positions on the walkway, 2) The edge of the single step created by the PAMELA platform was chamfered, and 3) The walkway had typical concrete grey colour.

\section{Conclusions}

Principal component analysis in this study demonstrated to evaluate pedestrian capability how to manage stepping over the single step and showed differences between young and older groups. These findings might be useful to consider how to improve outdoor and indoor environments as well as to assess how physical training, preventive measures, and assistive technologies to reduce the risk of tripping in stepping over the single step.

\section{References}

1. Bird, S.: The risk of tripping accidents on public footways. Proceedings of the Institution of Civil Engineers - Municipal Engineer 161, pp.129-136 (2008).

2. W.P. Berg, W.P., Alessio, H.M., Mills, E.M., and C. Tong, C.: 1997. Circumstances and consequences of falls in independent community-dwelling older adults. Age and ageing 26, pp.261-268 (1997)

3. Mindell, J.S., Leslie, D., and Wardlaw, M.: Exposure-based, "like-for-like" assessment of road safety by travel mode using routine health data. PLoS ONE 7(12), e50606 (2012).

4. Tinetti, M.E., Speechley, M., and Ginter, S.F.: Risk Factors for Falls among Elderly Persons Living in the Community. New England Journal of Medicine 319(26), pp.1701-1707 (1988)

5. Mills, P.M., Barrett, R.S. and Morrison, S.: Toe clearance variability during walking in young and elderly men. Gait \& Posture 28, pp.101-107 (2008)

6. Chen,H.-C., Ashton-Miller, J.A., Alexander, N.B. and A.B. Schultz, A.B.: Stepping Over Obstacles: Gait Patterns of Healthy Young and Old Adults. J Gerontol 46(6), pp. M196203. (1991)

7. Loverro, K.L., Mueske, N.M. and Hamel, K.A.: Location of minimum foot clearance on the shoe and with respect to the obstacle changes with locomotor task. Journal of Biomechanics 46, pp.1842-1850 (2013)

8. Uttley, J., Fotios, S., and Cheal, C.: Effect of illuminance and spectrum on peripheral obstacle detection by pedestrians. Lighting Research and Technology 49, pp.211-227 (2017)

9. Cheng, T.J., Yang, B., HOLLOWAY, C. and Tyler, N.: Effect of environmental factors on how older pedestrians detect an upcoming step. Lighting Research and Technology 50, pp.405-415 (2018)

10. K. Pearson, "On Lines and Planes of Closest Fit to Systems of Points in Space", Philosophical Magazine. 2 (11), pp.559-572, 1901 